\title{
Desenvolvimento e validação de tecnologia educativa para adolescentes com Diabetes
}

\section{Mellitus Tipo 1}

Development and validation of educational technology for adolescents with Type 1 Diabetes Mellitus Desarrollo y validación de tecnología educativa para adolescentes con Diabetes Mellitus Tipo 1

Talita Silva Rebouças

ORCID: https://orcid.org/0000-0002-8378-8803 Universidade Estadual do Ceará, Brasi E-mail: talitinhareboucas@hotmail.com Janaina dos Santos Mendes

ORCID: https://orcid.org/0000-0002-6200-6294 Universidade Estadual do Ceará, Brasil E-mail: janainasmendes22@gmail.com

Maria Eunice Nogueira Galeno Rodrigues ORCID: https://orcid.org/0000-0002-3245-3712 Universidade Estadual do Ceará, Brasil

E-mail: eunicegaleno@hotmail.com

Roselene Soares de Oliveira https://orcid.org/0000-0002-0067-7324 Universidade Estadual do Ceará, Brasil E-mail: rose.fisio@uol.com.br

Débora Silveira de Lima

ORCID: https://orcid.org/0000-0002-4841-2333 Universidade Estadual do Ceará, Brasi

E-mail: debora.s1001@gmail.com Ana Caroline Araujo da Silva ORCID: https://orcid.org/0000-0001-7864-8761 Universidade Estadual do Ceará, Brasil E-mail: anacaroline222324@gmail.com

Samila Sousa Vasconcelos ORCID: https://orcid.org/0000-0002-1509-1887

Centro Universitário Inta - Uninta, Brasil

E-mail: samilasousa@hotmail.com

Cláudia Patrícia da Silva Ribeiro Menezes

ORCID: https://orcid.org/0000-0002-0559-9302 Universidade Estadual do Ceará, Brasil E-mail: claudia_ribeiro6@hotmail.com

Lívia Lopes Custodio ORCID: https://orcid.org/0000-0001-9610-7379 Universidade Estadual do Ceará, Brasil E-mail: liviacustodio@yahoo.com.br

Leticia Alexandre Lima

ORCID: https://orcid.org/0000-0003-2566-0315 Universidade Estadual do Ceará, Brasi

E-mail: leticia_lima1@hotmail.com

Débora Pena Batista e Silva

ORCID: https://orcid.org/0000-0003-0633-5242 Universidade Estadual do Ceará, Brasil E-mail: pbs.debora@gmail.com

Débora Cristina Couto Oliveira Costa ORCID: https://orcid.org/0000-0002-9402-0613 Universidade Estadual do Ceará, Brasi

E-mail: deboraccoc@gmail.com

Ilvana Lima Verde Gomes

ORCID: https://orcid.org/0000-0002-1861-5121 Universidade Estadual do Ceará, Brasil E-mail: ilverde@gmail.com

\section{Resumo}

A Diabetes Mellitus tipo 1 (DM tipo 1) é uma doença crônica de caráter multifatorial, que pode atingir qualquer idade, sendo mais comum em crianças, adolescentes e jovens. A DM pode ser administrada através de quatro pilares básicos: 
insulino-terapia, dietoterapia , monitorização do controle glicêmico e exercício físico. A educação em saúde é uma importante ferramenta que pode ser usada para contribuir com alguns desses pilares. Objetivou-se desenvolver uma tecnologia educativa para adolescentes com DM tipo 1. Trata-se de um estudo metodológico. Os passos foram: 1) Aprovação do projeto no Comitê de Ética em Pesquisa; 2) Revisão de literatura e documental; 3) Seleção do Conteúdo; 4) Construção da história em quadrinho; 5) Validação interna.Na análise estatística,o Índice de Validade de Conteúdo (IVC) global da tecnologia educativa foi de $95 \%$ entre juízes de conteúdo e juízes técnicos. Entre os especialistas de design e marketing, a tecnologia educativa foi considerada como material superior, 94,61\%. A avaliação dos juízes de conteúdo, classificou a tecnologia educativa validada com um IVC de 0,96. O IVC da tecnologia educativa foi de 1,0, ratificando a validação da sua aparência e conteúdo junto ao grupo de juízes técnicos e de propaganda e marketing. Desse modo, a cartilha educativa constitui material válido e confiável que pode ser utilizado como ferramenta na educação em saúde e servir como fonte de guia para os adolescentes que vivem com diabetes tipo 1, promovendo a adesão e o autogerenciamento do tratamento.

Palavras-chave: Diabetes mellitus tipo 1; Adolescente; Tecnologia educacional.

\begin{abstract}
Type 1 Diabetes Mellitus (Type $1 \mathrm{DM}$ ) is a chronic disease of multifactorial character, which can reach any age, being more common in children, adolescents and young people. DM can be administered through four basic pillars: insulin therapy, diet therapy, monitoring glycemic control and physical exercise. Health education is an important tool that can be used to contribute to some of these pillars. The objective was to develop an educational technology for adolescents with type $1 \mathrm{DM}$. This is a methodological study. The steps were: 1) Approval of the project by the Research Ethics Committee; 2) Literature and document review; 3) Content selection; 4) Construction of the story in a comic; 5) Internal validation. In the statistical analysis, the global Content Validity Index (CVI) of educational technology was $95 \%$ between content judges and technical judges. Among design and marketing specialists, educational technology was considered to be superior material, 94.61\%. The evaluation of the content judges, classified the educational technology validated with an IVC of 0.96 . The CVI of educational technology was 1.0, confirming the validation of its appearance and content with the group of technical and advertising and marketing judges. Thus, the educational booklet is valid and reliable material that can be used as a tool in health education and serve as a source of guidance for adolescents living with type 1 diabetes, promoting adherence and self-management of treatment.
\end{abstract}

Keywords: Diabetes mellitus type 1; Adolescent; Educational technology.

\title{
Resumen
}

La Diabetes Mellitus Tipo 1 (DM Tipo 1) es una enfermedad crónica de carácter multifactorial, que puede llegar a cualquier edad, siendo más común en niños, adolescentes y jóvenes. La DM se puede administrar a través de cuatro pilares básicos: terapia con insulina, dietoterapia, monitorización del control glucémico y ejercicio físico. La educación sanitaria es una herramienta importante que puede utilizarse para contribuir a algunos de estos pilares. El objetivo fue desarrollar una tecnología educativa para adolescentes con DM tipo 1. Se trata de un estudio metodológico. Los pasos fueron: 1) Aprobación del proyecto por parte del Comité de Ética en Investigación; 2) Revisión de literatura y documentos; 3) Selección de contenido; 4) Construcción de la historia en un cómic; 5) Validación interna En el análisis estadístico, el Índice de Validez de Contenido (IVC) global de tecnología educativa fue del $95 \%$ entre jueces de contenido y jueces técnicos. Entre los especialistas en diseño y marketing, la tecnología educativa fue considerada como material superior, 94,61\%. La evaluación de los jueces de contenido, clasificó la tecnología educativa validada con un IVC de 0.96. El CVI de tecnología educativa fue 1.0, lo que confirma la validación de su apariencia y contenido con el grupo de jueces técnicos y publicitarios y de marketing. Así, el folleto educativo es un material válido y confiable que se puede utilizar como herramienta en la educación para la salud y servir como fuente de orientación para los adolescentes que viven con diabetes tipo 1, promoviendo la adherencia y el autocontrol del tratamiento.

Palabras clave: Diabetes mellitus tipo 1; Adolescente; Tecnología educacional.

\section{Introdução}

Diabetes mellitus (DM) apresenta-se como uma das doenças crônicas não transmissíveis (DCNT) de maior prevalência e incidência na população. É classificada como uma disfunção metabólica, com hiperglicemia persistente devido a deficiência na produção de insulina e pode ser classificada em três tipos principais: Diabetes tipo I, tipo II e gestacional (Lima Filho et al., 2020). Considera-se que o DM não seja uma única doença, mas um grupo heterogêneo de distúrbios metabólicos que apresenta em comum a característica de defeitos na ação da insulina, na sua secreção ou em ambas, capaz de desencadear mudanças significativas na qualidade de vida dos pacientes (Sociedade Brasileira de Diabetes - SBD, 2019).

A Organização Mundial da Saúde (OMS) prevê para o ano de 2030 um quantitativo de aproximadamente 11,3 
milhões de pacientes com diabetes, isso somente no Brasil. Caso haja a confirmação dessa previsão, ter-se-á como consequência a sobrecarga do Sistema Único de Saúde, que no período de 2010 a 2016 teve um gasto de aproximadamente 2,6 milhões com pacientes diabéticos e renais crônicos (Nilson et al., 2020; Salin et al., 2019).

O diabetes mellitus tipo 1 (DM1) é uma doença crônica autoimune de caráter multifatorial que pode acometer diferentes faixas etárias, sendo mais comumente diagnosticada em crianças, adolescentes e adultos jovens. Apesar da idade inicial ser preponderante para o desenvolvimento sintomático durante a infância ou adolescência, os sintomas podem ser surgirem tardiamente. Deste modo, corresponde acerca de 5-10\% dos casos de diabetes e caracteriza-se pela destruição progressiva e insidiosa das células $\beta$ produtoras de insulina das ilhotas pancreáticas, usualmente desencadeando a deficiência absoluta de insulina. Requer cuidados médicos contínuos e de educação em saúde para o auto manejo do controle glicêmico com vistas a prevenir complicações agudas e reduzir o risco de complicações crônicas (Katsarou et al, 2017; SBD, 2019).

Segundo publicação do Atlas da Internacional Diabetes Federation (IDF) (2019) a estimativa é que 1,1 milhão de crianças e adolescentes (com menos de 20 anos) tenham diabetes tipo 1 . O acometimento do diabetes tipo 1 apresenta uma significativa variação geográfica, revelando taxas por 100 mil indivíduos com menos de 15 anos de idade, a incidência no Brasil é de 7,6. Com isso, observa-se o aumentando desordenado nas últimas décadas da incidência de diabetes tipo 1, especificamente entre crianças com menos de 5 anos de idade. Em 2017, a estimativa mundial da DM1, correspondente à faixa etária de 0 a 19 anos, foi de 1.104.500, com estimativa de surgimento de 132 mil casos novos por ano (SBD, 2019).

$\mathrm{Na}$ assistência às pessoas com DM, a educação em saúde é apontada como uma estratégia que deve ser resgatada e valorizada como um instrumento de trabalho fundamental, levando em consideração todas as características da doença e as demandas geradas por elas, para um controle efetivo e integral, que seja capaz de influenciar na prevenção das complicações crônicas (Salci; Meirelles \& Silva, 2018).

A educação em saúde é uma importante ferramenta pois interfere na melhoria na qualidade de vida dos pacientes uma vez que esta prática é capaz de modificar os estilos de vida dos indivíduos e das comunidades (Mallmann et al, 2015). Uma estratégia utilizada visando o objetivo da educação em saúde é o uso de materiais educativos, que atuam como instrumentos no intermédio entre políticas e práticas de saúde enfatizando os discursos e procedimentos médico-sanitários, o diagnóstico, o tratamento, a vigilância de casos e a relação profissional de saúde-paciente (Massara et al, 2016).

Para que as tecnologias sejam ferramentas efetivas para serem usadas no cuidado e na disseminação do conhecimento, é fundamental que a informação seja transmitida de maneira eficiente, devendo ser elaborada da maneira mais clara possível para o bom entendimento. Assim, faz-se necessário o desenvolvimento de estratégias educativas claras e de fácil compreensão para abranger um público maior e com a certeza de que o conhecimento foi assimilado (Dodt, 2011).

Desta forma, surgiu o interesse por criar e validar uma história em quadrinhos, que se faz relevante, pois a tecnologia proposta promoverá ações educativas na melhoria do tratamento e promoção do autocuidado, além de possibilitar uma aplicabilidade no serviço, direcionando suas ações mais adequadamente para atingir resultados satisfatórios. O objetivo desta pesquisa foi, portanto, desenvolver e validar uma tecnologia educativa para adolescentes com DM tipo 1.

\section{Metodologia}

Trata-se de um estudo de validação de tecnologia do tipo de pesquisa de desenvolvimento metodológico (Nascimento, 2012) com abordagem qualitativa (Bardin, 2011), que se refere à construção e validação de um instrumento de pesquisa. A pesquisa teve como critério metodológico desenvolver duas fases intituladas como: $1^{\mathrm{a}}$ Fase: Diagnóstico Situacional e $2^{\mathrm{a}}$ Fase: Construção e Validação Interna da Tecnologia Educativa. 


\subsection{Primeira Fase: Diagnóstico Situacional}

Esta fase da pesquisa, tem como fundamental importância mapear o cenário de pesquisa e embasar, juntamente com a revisão integrativa realizada posteriormente, a construção da tecnologia educativa.

Para a elaboração do diagnóstico situacional foi utilizado como cenário, o ambulatório de Endocrinologia, um hospital de referência na atenção terciária do SUS situado em Fortaleza-Ceará. Neste momento utilizou-se como recurso metodológico, aplicação de questionário com os adolescentes com DM tipo 1. As dúvidas dos adolescentes entrevistados sobre o tema foram incluídas na história em quadrinhos com objetivo de obter conteúdo para o desenvolvimento da tecnologia educativa. A pesquisadora selecionou 20 participantes, sendo utilizada uma amostragem por conveniência. Esse número justifica - se, pois, esses adolescentes apresentam assiduidade nas consultas. E para critérios de inclusão, definiu-se: adolescentes com DM tipo 1, com idade entre 10 a 19 anos de acordo com a OMS (WHO, 2015), serem conscientes e orientados. Já como critérios de exclusão, estabeleceu-se: os participantes que não comparecessem às consultas no ambulatório de Endocrinologia.

A fim de alcançar esses dados iniciais, foram realizadas entrevistas semiestruturadas, que eram realizadas antes ou após as consultas, da maneira mais cômoda para os adolescentes e para os responsáveis. As entrevistas foram no período de maio a julho de 2017 de segunda a sexta pela manhã.

O roteiro de entrevista foi composto por quatros perguntas de caracterização do participante e seis perguntas norteadoras sobre o objeto em estudo. As entrevistas foram gravadas. $\mathrm{O}$ tempo de entrevista variou de cinco a dez minutos.

O material transcrito na íntegra foi organizado em arquivos individuais e, para a sua análise, foram seguidas as etapas recomendadas pela Análise Categorial Temática de Bardin (2011): pré-análise; exploração do material; tratamento dos resultados, a inferência e interpretação.

\subsection{Segunda Fase: Construção e Validação Interna da Tecnologia Educativa}

Para o desenvolvimento dessa etapa de construção e validação da tecnologia educativa, foram seguidos os passos embasados nas recomendações de Echer (2005), que têm sido referência em diversas pesquisas na orientação acerca de como desenvolver manuais de educação em saúde. De forma sintetizada, os passos foram: 1. Aprovação do projeto no Comitê de Ética em Pesquisa; 2. Revisão de literatura e documental; 3. Seleção do Conteúdo; 4. Construção da história em quadrinho; 5. Validação interna.

No que condiz ao $1^{\circ}$ passo, ocorreu antes da primeira fase, seguindo o rigor ético da pesquisa. A pesquisa foi iniciada após a aprovação no Comitê de Ética em Pesquisa (CEP) do Hospital Geral de Fortaleza / SUS sob parecer de no 1.912.551. Destaca-se que foram preconizadas as normas da Resolução 466/2012 do Conselho Nacional de Saúde (CONEP) (Brasil, 2013), que determina o respeito à autonomia dos sujeitos, a beneficência, a não-maleficência e a justiça. Portanto, foi entregue aos juízes e familiares o Termo de Consentimento Livre e Esclarecido e para os adolescentes um Termo de Assentimento Livre e Esclarecido.

Realizou-se no $2^{\circ}$ passo, uma busca na literatura, tendo como critérios de inclusão, as publicações de temáticas relacionadas à diabetes mellitus tipo $1 \mathrm{em}$ adolescentes, nos últimos 15 anos, incluindo publicações completas com resumos disponíveis eletronicamente nos idiomas inglês e português. Os critérios de exclusão foram trabalhos do tipo monografia, teses e dissertações e trabalhos publicados antes do ano 2000.

Quanto ao $3^{\circ}$ passo correspondente a seleção do conteúdo foi realizada em maio de 2016 através do acesso on-line em bases de dados importantes no contexto da saúde indexados nas bases Directory of Open Access Journals (DOAJ), National Library of Medicine (Medline/PubMed); Literatura Latino-Americana e do Caribe em Ciências da Saúde (LILACS); Scientific Electronic Library Online (SCIELO) e Scopus (Elsevier). Realizou-se o entrecruzamento dos seguintes descritores controlados, presentes no DeCs/Mesh (Descritores em Ciências da Saúde/Medical Subject Heading): diabetes mellitus tipo 1 e adolescente 
(português e inglês) e o marcador booleano "AND”.

Para o $4^{\circ}$ passo intitulado como construção da história em quadrinho, utilizou-se uma designer gráfica, que participou da elaboração da arte da história em quadrinhos. A designer elaborou essa arte a partir de um roteiro produzido pela pesquisadora, mas com as falas dos participantes entrevistados. Os personagens e os nomes foram dos membros da família da pesquisadora. A design enviava os desenhos para aprovação da pesquisadora e alterações foram feitas para a confecção do material. O Adobe ilustrador CS3 foi o programa para os desenhos e Adobe In design CS6 para diagramação. As fontes foram "Komika Text Kaps" tamanho 12 para o texto geral, tamanho 33 para subtítulo da capa e "Kraash Black" tamanho 45 para o título da capa e algumas onomatopéias. Esse passo da pesquisa, utilizou como base o diagnóstico situacional realizado, a revisão da literatura e as Diretrizes da Sociedade Brasileira de Diabetes.

Em relação ao $5^{\circ}$ passo pertinente ao processo de validação interna, Teixeira, Nascimento e Medeiros (2014) falam acerca da necessidade das tecnologias impressas elaboradas serem submetidas a análise de pessoas com grande conhecimento e experiência na área (juízes-especialistas): 1. Juízes Especialistas (doutores com alto grau de conhecimento sobre o conteúdo do Diabetes Melitus tipo 1); 2. Juízes Técnicos (profissionais e trabalhadores da saúde com experiência assistencial ou de educação em saúde na temática Diabetes Mellitus tipo 1); 3. Juízes de Comunicação (profissionais com experiência em comunicação social e/ou design gráfico). Procurou-se esses juízes de comunicação em empresas que fornecem serviços de publicidade e propaganda, marketing e comunicação.

Nesta perspectiva, a validação seguiu os passos de Pasquali (1997): seis a vinte, totalizando a participação de juízes. O autor orienta que o número de juízes de seis a vinte especialistas é o recomendável para a validação, com, no mínimo, de três indivíduos que podem ser divididos em grupos de especialistas. Dividiu-se os juízes em três grupos distintos: grupo de juízes de conteúdo (seis pesquisadores docentes), grupo de juízes técnicos (dois enfermeiros com experiência em diabetes tipo 1 com adolescentes) e grupo de juízes com experiência profissional em propaganda e marketing (três) para a validade da aparência.

Utilizou-se a estratégia de amostragem do tipo "bola de neve" (denominada de amostra em rede) (Dewes, 2013), no qual após escolha do primeiro juiz, este indica outro profissional que se enquadre na pesquisa e assim, sucessivamente, sendo todos avaliados quanto aos critérios de inclusão após serem indicados (Polit; Beck, 2011). O contato foi feito por meio do envio de e-mails, com a carta convite contendo os objetivos e descrição da pesquisa. Após o aceite, era enviado o material com a história em quadrinhos, o Instrumento de Avaliação da Tecnologia Educativa e o Termo de Consentimento Livre e Esclarecido, e solicitava-se a análise, avaliação e validação da tecnologia.

Realizou-se convites de janeiro a fevereiro de 2018. No total 21 pessoas foram convidadas para participar da pesquisa, mas nem todas aceitaram. Obteve-se, portanto, seis especialistas, dois técnicos e três de comunicação. A pesquisadora forneceu, inicialmente, um prazo de 15 dias para que cada juiz realizasse a sua avaliação.

Os juízes especialistas e os juízes técnicos utilizaram um instrumento relacionado ao conteúdo e a aparência da tecnologia educativa elaborada. Este era composto de itens acerca de todo o conteúdo abordado no material criado a partir de uma escala de avaliação do tipo Likert, que possuía uma pontuação de um a quatro, com base nas opiniões dos juízes: (4) totalmente adequado, (3) adequado, (2) parcialmente adequado, (1) inadequado. Ao final da escala de avaliação, existia um espaço para que os juízes pudessem expressar suas opiniões e sugestões quanto ao material elaborado.

Em relação à validade do conteúdo a partir dessa avaliação, foi utilizado o Índice de Validação de Conteúdo (IVC) proposto por Waltz e Baussel (1981).

A partir desse processo de avaliação, foi possível obter-se uma tecnologia devidamente validada quanto ao seu conteúdo, sua aparência e a semântica. Logo após a criação da versão final, foi realizada a impressão de exemplares. 


\section{Resultados e Discussão}

Os resultados estão apresentados em três etapas, de acordo com os objetivos deste estudo. Na primeira etapa, descreveu-se os resultados em relação ao processo de construção da história em quadrinho; na segunda etapa, a validação de conteúdo e aparência; e na terceira etapa, os resultados relativos à implementação da cartilha com os adolescentes.

\subsection{Descrição Da História Em Quadrinhos}

A História em quadrinhos foi intitulada "Diabetes não é brinquedo" inicialmente tinha 25 páginas com a vivência dos adolescentes com diabetes. Os personagens foram os membros da família da pesquisadora. Esse nome da história em quadrinhos foi para ilustrar que diabetes é um desafio e precisa ser encarada com seriedade. A paciente Talita foi construída com o mesmo nome e a semelhança da aparência da pesquisadora também.

Nessa primeira fase para construção da História em quadrinhos foi subsidiada pela pesquisa na literatura, entrevistas com adolescente e diretrizes da Sociedade Brasileira de Diabetes. As falas dos personagens foram elaboradas a partir das dúvidas dos adolescentes coletadas durante as entrevistas. A construção da História em quadrinhos através dessas falas relatadas pelos adolescentes entrevistados trouxe um enfoque lúdico para as experiências vivenciadas por eles.

O Diabetes foi definido pelos adolescentes ao serem questionados durante entrevistas. Nesta pesquisa, optou-se por usar nomes de frutas para não identificar os adolescentes. Esses nomes de frutas no masculino e feminino foram para diferenciar o sexo do participante:

[...] "Diabetes é uma doença que a pessoa pode viver normal controlando ela. As pessoas sentem algumas coisas quando ela está baixa, cansaço nas pernas, fraco, suor gelado, mas controlando ela pode ter uma vida normal. Pode participar de jogos, jogar e outras coisas" (Pêssego).

[...] "Eu estava provocando e não estava querendo comer. Deram injeção para vômito só que não serviu. Aí nós viemos de novo para o hospital. Ai fiquei no soro. Ai me colocaram para outro hospital. Ai descobriram” (Mamão).

[...] "A minha mãe estava me achando triste, um pouquinho deprimido. Aí a tia dela me mandou para o médico, para o hospital. Aí ela furou meu dedo e colocou lá na máquina e deu trezentos e pouco" (Kiwi).

Dessa forma, inicia-se a história em quadrinhos com a indagação que foi feita durante as entrevistas com os adolescentes: "Como descobriu diabetes tipo 1?" Foram adaptadas as falas dos participantes "pêssego", "mamão" e "kiwi". Nesses relatos, fica claro a variedade do processo de descobrimento da doença para cada participante, optando-se, portanto, por descrever, na história em quadrinhos, a fisiopatologia do diabetes a partir de pesquisa na literatura.

A história em quadrinhos teve como intuito promover educação em saúde para os adolescentes com DM1. Esse recurso lúdico utiliza muitas figuras para instigar o público adolescente à leitura do material construído.

Optou-se por dividir a história em quadrinhos em nove domínios, a partir das dúvidas principais identificados no diagnóstico situacional e nos temas mais frequentes que emergiram na busca feita na literatura (Haller; Atkinson \& Schatz, 2005; Luna et al, 2005; Orbey et al, 2007; Pace; Nuces \& Ochoa-Vigo, 2003; Queiroz; Silva \& Alfenas, 2010; SBF, 2015).

"Como Talita descobriu diabetes tipo 1...": Neste domínio foi abordado na consulta médica a fisiopatologia e os sinais e sintomas da diabetes tipo 1.

“Talita vai à consulta de Enfermagem...": A enfermeira abordada na consulta de Enfermagem a temática hipoglicemia e hiperglicemia.

“Talita aprendendo a aplicar insulina...”: A enfermeira orienta a aplicação da insulina com seringa e o descarte dela. 
"Talita aprendendo a aplicar insulina com caneta...": Na consulta de Enfermagem, a enfermeira orienta o uso da insulina na caneta e o armazenamento.

“Talita aprendendo os locais de aplicação...”: A orientação sobre os locais adequados de aplicação de insulina.

"Talita e a dieta...": A enfermeira reúne os adolescentes num piquenique educativo para tirar as dúvidas sobre os alimentos.

“Talita aprendendo contagem de carboidrato...": A nutricionista faz orientações acerca da contagem de carboidrato.

"Talita fitness...": Incentiva o adolescente com diabetes tipo 1 a prática regular de exercício físico.

"Dicas de exercícios! ”: As dicas de exercício em relação a administração de insulina e ingestão de carboidrato.

\subsection{Validação Da Tecnologia Educativa}

A história em quadrinhos construída submeteu-se à validação por onze juízes especialistas, sendo oito enfermeiros que atuam na área de diabetes melitus tipo 1, tecnologias educativas e/ou validação de instrumentos e três da área de designer e marketing.

\subsubsection{Validação Por Juízes Especialistas}

Os juízes de conteúdo que validaram a história em quadrinhos foram seis. Todos enfermeiros, doutores e docentes.

A maioria dos juízes avaliou os objetivos da tecnologia educativa como "adequado" ou "totalmente adequado", o que conferiu um IVC de 0,89 para os objetivos propostos. Em relação ao item 1.2 "Promove mudança de comportamento e atitude em relação ao cuidado em saúde", um juiz avaliou como "parcialmente adequado", pois apenas saberá se incentiva a mudança em um estudo clínico. Uma juíza avaliou esse item como "não se aplica", sendo este critério revisado.

Os juízes classificaram estrutura e apresentação como 0,99. No item, "As mensagens estão apresentadas de maneira clara e objetiva", uma juíza avaliou como parcialmente adequada ou não se aplica.

$\mathrm{Na}$ avaliação da relevância, nenhum item foi julgado inadequado ou marcado como "não se aplica". Verificou-se que todos os itens foram validados como "adequado" ou "totalmente adequado", o que conferiu um IVC de 1,00 para os objetivos propostos.

Os resultados expostos revelam que a avaliação dos juízes de conteúdo, classificou a tecnologia educativa validada com um IVC de 0,96. As taxas de concordância consideradas aceitáveis são variáveis e a literatura recomenda que sejam entre 70-100\% (Teixeira; Nascimento \& Medeiros, 2014), sendo que nessa pesquisa foi utilizada a de valores maiores ou iguais a 0,78 (IVC >0,78), por ser considerado um valor que revela boa qualidade do material elaborado (Alexandre \& Coluci, 2011).

\subsubsection{Validação Por Juízes Técnicos}

$\mathrm{Na}$ avaliação dos juízes técnico, os itens foram validados tendo em vista que o IVC foi de 0,87 . Todos os juízes consideraram adequado ou totalmente adequado. Nenhum juiz avaliou como "inadequado". O processo de avaliação deste grupo de juízes, tanto a maneira como a sequência, foi semelhante ao grupo de juízes de conteúdo.

$\mathrm{Na}$ avaliação realizada pelos juízes técnicos, quanto aos objetivos da tecnologia educativa, nenhum item foi julgado "inadequado" ou marcado como "não se aplica". A maioria dos juízes especificou como "adequado" ou "totalmente adequado", confirmando para os objetivos propostos um IVC de 1,0.

Nenhum item foi julgado "inadequado", "parcialmente adequado" ou marcado como NA (não se aplica). No quesito relevância, foi conferido à cartilha o IVC de 1,00, pois os juízes consideraram todos os itens como "adequado" ou "totalmente adequado".

Portanto, o IVC da tecnologia educativa foi de 1,0, ratificando a validação da sua aparência e conteúdo junto ao grupo 
de juízes técnicos.

Os resultados revelam que a avaliação dos juízes de conteúdo e técnicos, classificou a tecnologia educativa como validada.

\subsubsection{Validação Por Juízes Especialistas Da Área De Propaganda E Marketing}

Após validação, a história em quadrinhos constitui de 26 páginas. O formato da história foi A4 ( $29,7 \mathrm{~cm}$ x $21 \mathrm{~cm})$. As fontes foram "Komika Text Kaps" tamanho 12 para o texto geral, tamanho 33 para subtítulo da capa e "Kraash Black" tamanho 45 para o título da capa e algumas onomatopeias (Figura 1).

Figura 1: Versão pré-validação; versão pós-validação da capa da história em quadrinhos.
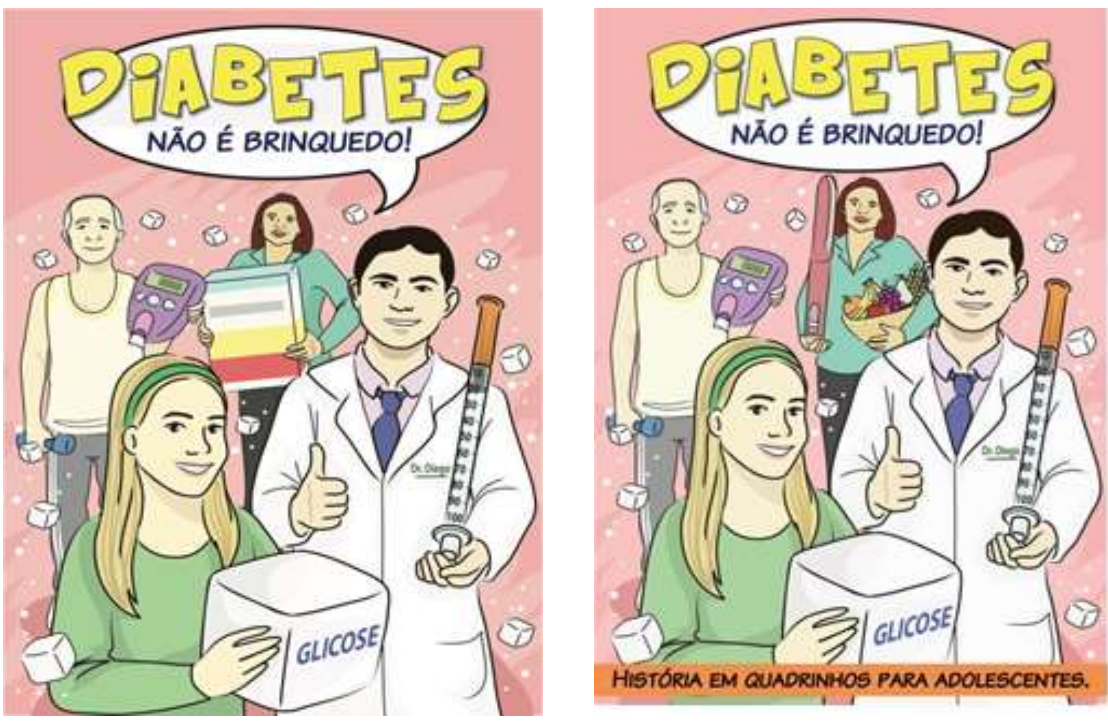

Fonte: Autoria própria: Rebouças et al, (2016) - Tecnologia educativa: "Diabetes não é brinquedo! - História em quadrinhos para adolescente." Elaborada em 2016.

Na Figura 1, observa-se as duas versões da capa, pré-validação e pós validação, na segunda versão, enfatizou-se o público alvo da história em quadrinho. A capa foi elaborada com os personagens referentes a família da pesquisadora e a pesquisadora como paciente com DM1. Após a validação, os juízes sugeriram que deixasse claro que a história em quadrinhos "Diabetes não é brinquedo" era para adolescentes e no prefácio também teria que deixar claro. Uma juíza relatou que deveria retirar a caixa de medicação, pois o tratamento do diabetes tipo 1 é com insulina. Portanto, essa caixa de medicação da capa inicial foi retirada e substituída por caneta de insulina e acrescentadas frutas nas ilustrações para contemplar as temáticas abordadas na tecnologia educativa.

A história em quadrinhos inicia com a apresentação do profissional médico Dr. Diego, e da Talita, paciente adolescente com DM 1. Após a validação, as juízas sugeriram uma mudança do título "Como Talita descobriu a diabetes tipo 1?” por “Como Talita descobriu diabetes tipo 1...”. Uma juíza técnica enfatizou que diabetes deveria ser no masculino, preferencialmente sem artigo definido. As reticências ficariam mais adequadas que o ponto de interrogação. Além disso, o termo "provocar", obtido a partir da fala do adolescente durante a entrevista, pelo termo "vomitar". Uma juíza técnica sugeriu que o diagnóstico médico ter sido dado apenas depois que a paciente relatou os sinais e sintomas durante a consulta. Na versão inicial, essa juíza técnica considerou incoerente que a paciente já citou que tinha DM1 durante o início dessa consulta, pois na prática não é comum um adolescente chegar conhecendo a classificação do diabetes.

A sexta página da tecnologia educativa (Figura 2) foi composta pela definição e explicação sobre a DM tipo 1. 
Figura 2: Versão pré e pós-validação da definição da DM tipo 1 na história em quadrinhos.
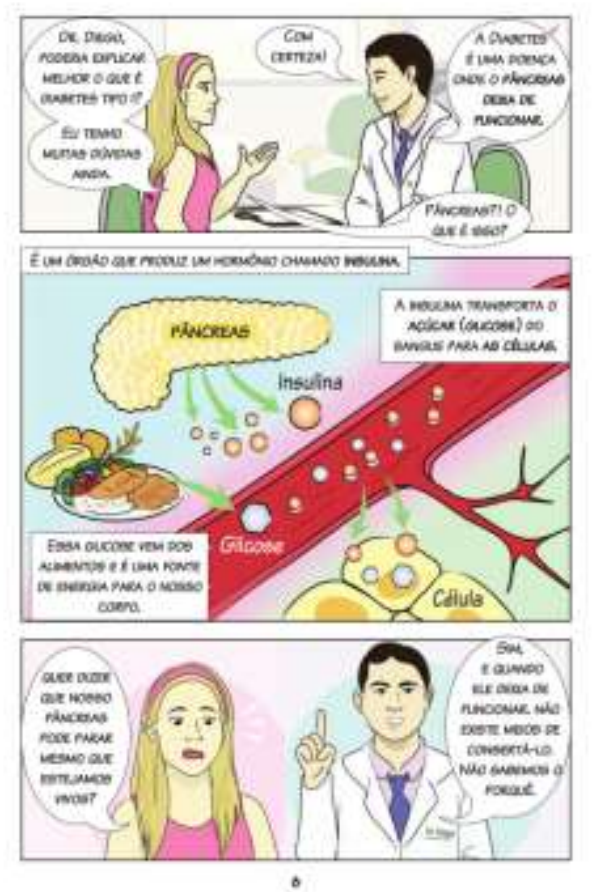
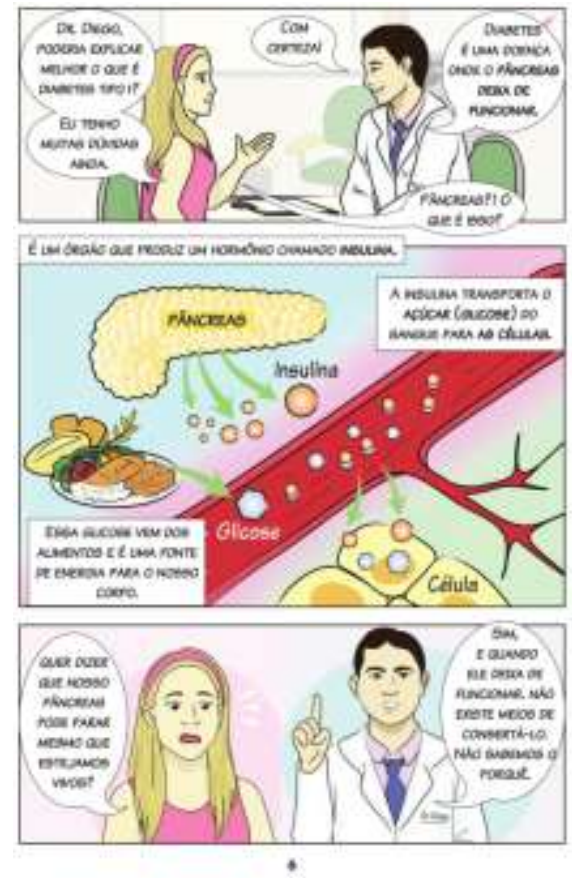

Fonte: Autoria própria: Rebouças et al, (2016) - Tecnologia educativa: "Diabetes não é brinquedo! - História em quadrinhos para adolescente." Elaborada em 2016.

Na Figura 2, observa-se a definição da DM tipo 1 por meio do diálogo entre a personagem Talita e o médico. A paciente Talita expõe suas dúvidas e o médico explica a Fisiologia envolvida na DM. As juízas não sugeriram mudanças nas ilustrações e nem no conteúdo após a validação.

Após validação, as juízas sugeriram que retirasse do material o artigo definido "a" em referência à diabetes. Acrescentaram também o termo "aí temos" e o termo adulto jovem. Avaliaram como confuso a seta do pâncreas a insulina da versão inicial. Sugerido acrescentar adultos jovens no quadro "a diabetes tipo 1 é mais comum em crianças e em adolescentes". A frase "Como o corpo não produz mais" pelo "pâncreas não produz mais". No último diálogo entre Talita e Dr. Diego, os dois últimos balões das falas foram considerados confusos na versão inicial e modificados para tornarem mais claro ao leitor.

A paciente Talita vai à consulta de Enfermagem acompanhada dos pais. Surge uma nova personagem na história em quadrinhos, a enfermeira Bete. Ela não foi homenagem aos membros da família e o nome foi pensado aleatório devido ao nome Diabetes. Acrescentadas em todas as ilustrações a identificação da categoria Enfermeira no jaleco da profissional após validação. Tanto a ilustração quanto o nome dos pais coincidem com os da pesquisadora. Nessa temática da Consulta de Enfermagem, os juízes solicitaram que DM1 fosse retirada entre parênteses. Quando a enfermeira citou "vou tirar suas dúvidas", uma juíza avaliou que seria mais adequado incluir na primeira pessoa do plural, pois daria ideia de equipe multiprofissional.

Durante a consulta de Enfermagem, a paciente teve orientações sobre diabetes tipo 1. A hipoglicemia e hiperglicemia foram bastante citadas nas falas durante as entrevistas como dúvida na rotina dos pacientes entrevistados. A enfermeira orienta sobre sinais e sintomas e conduta de emergência quando ocorrer hipoglicemia. Após a validação, uma juíza sugeriu que substituísse o termo "bombom". De acordo com a Sociedade Brasileira de Diabetes descreve "balas macias". Além disso, bombom em outras regiões é chocolate que poderia causar confusão na interpretação, visto que os chocolates são contraindicados nas hipoglicemias devido ao excesso de gordura (Figura 3). 
Figura 3: Versão pré e pós-validação dos sinais e sintomas da DM tipo 1 na história em quadrinhos.
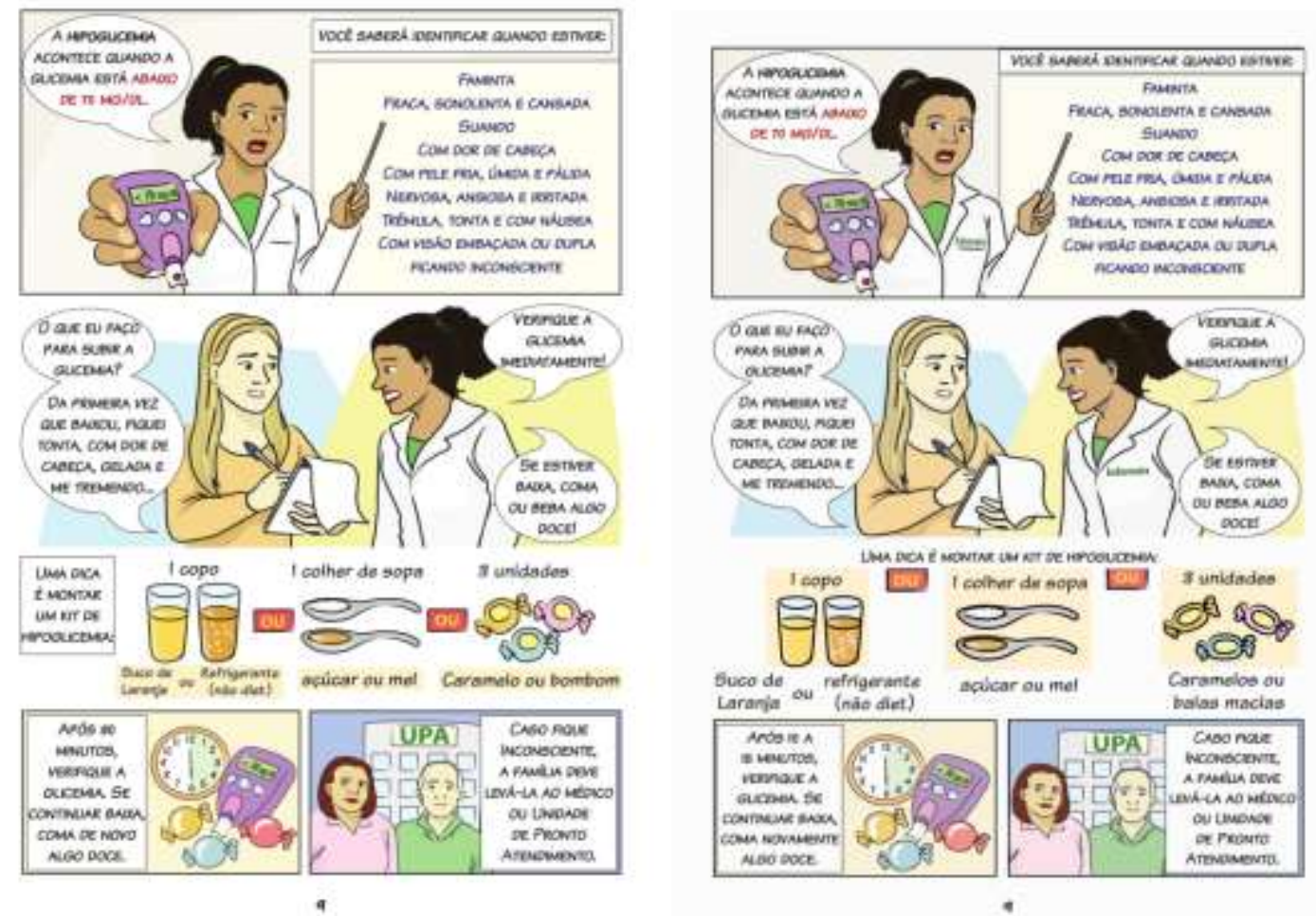

Fonte: Autoria própria: Rebouças et al, (2016) - Tecnologia educativa: "Diabetes não é brinquedo! - História em quadrinhos para adolescente." Elaborada em 2016.

A temática hiperglicemia foi abordada e uma juíza corrigiu os valores da hiperglicemia que na versão inicial era acima ou igual a $126 \mathrm{mg} / \mathrm{dl}$, no entanto esse é o ponto de corte para fechar o diagnóstico de diabetes. Hiperglicemia quando está acima de 180 a $220 \mathrm{mg} / \mathrm{dl}$. Outra juíza sugeriu que modificasse "já aprendi tudo sobre" por "já aprendi muito sobre", considerando que o aprendizado é contínuo (Figura 4). 
Research, Society and Development, v. 10, n. 5, e34710515087, 2021

(CC BY 4.0) | ISSN 2525-3409 | DOI: http://dx.doi.org/10.33448/rsd-v10i5.15087

Figura 4: Versão pré e pós-validação sobre dos valores de referência da hiperglicemia na história em quadrinhos.
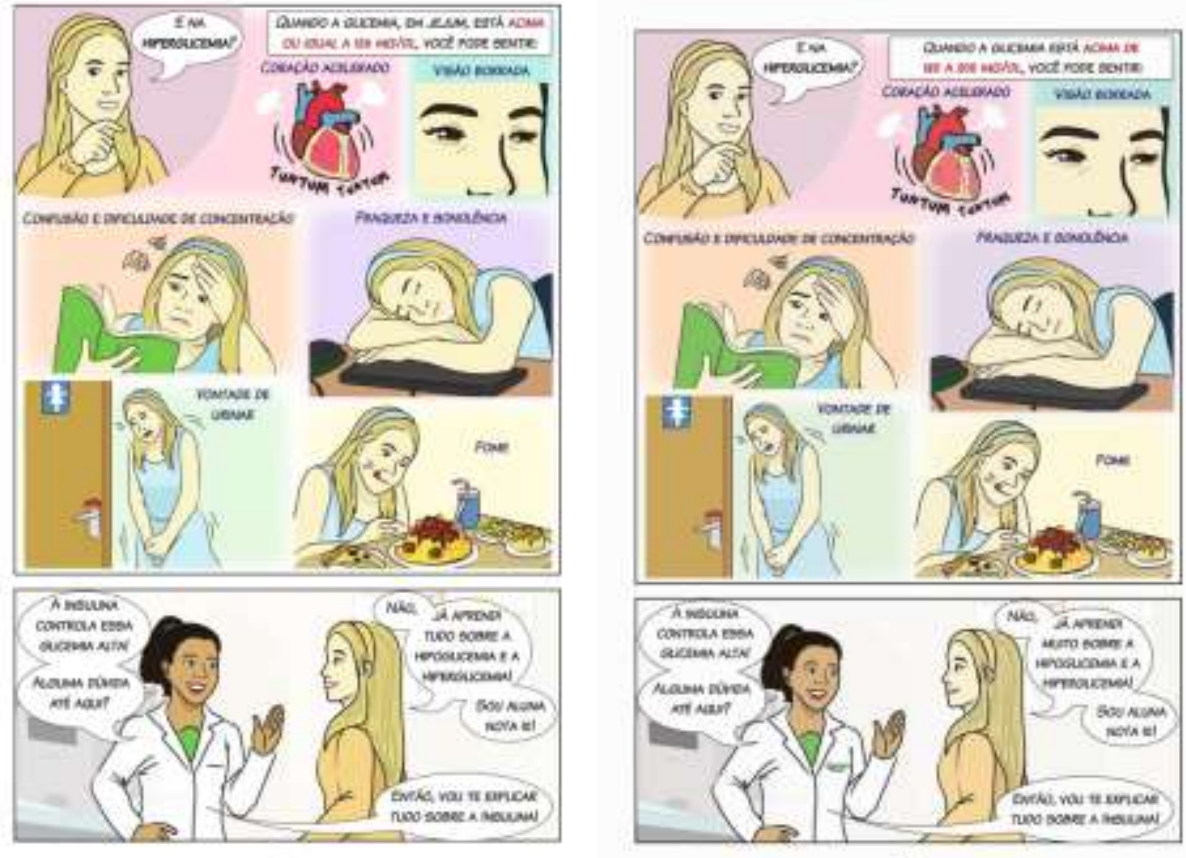

由

Fonte: Autoria própria: Rebouças et al, (2016) - Tecnologia educativa: "Diabetes não é brinquedo! - História em quadrinhos para adolescente." Elaborada em 2016.

O próximo assunto abordado foi a forma correta de aplicação de insulina (Figura 5), feito por meio do diálogo entre Talita e a enfermeira Bete. 
Figura 5: Versão pré-validação e pós-validação da forma correta de aplicação da insulina nos pacientes com DM tipo 1 na história em quadrinhos.
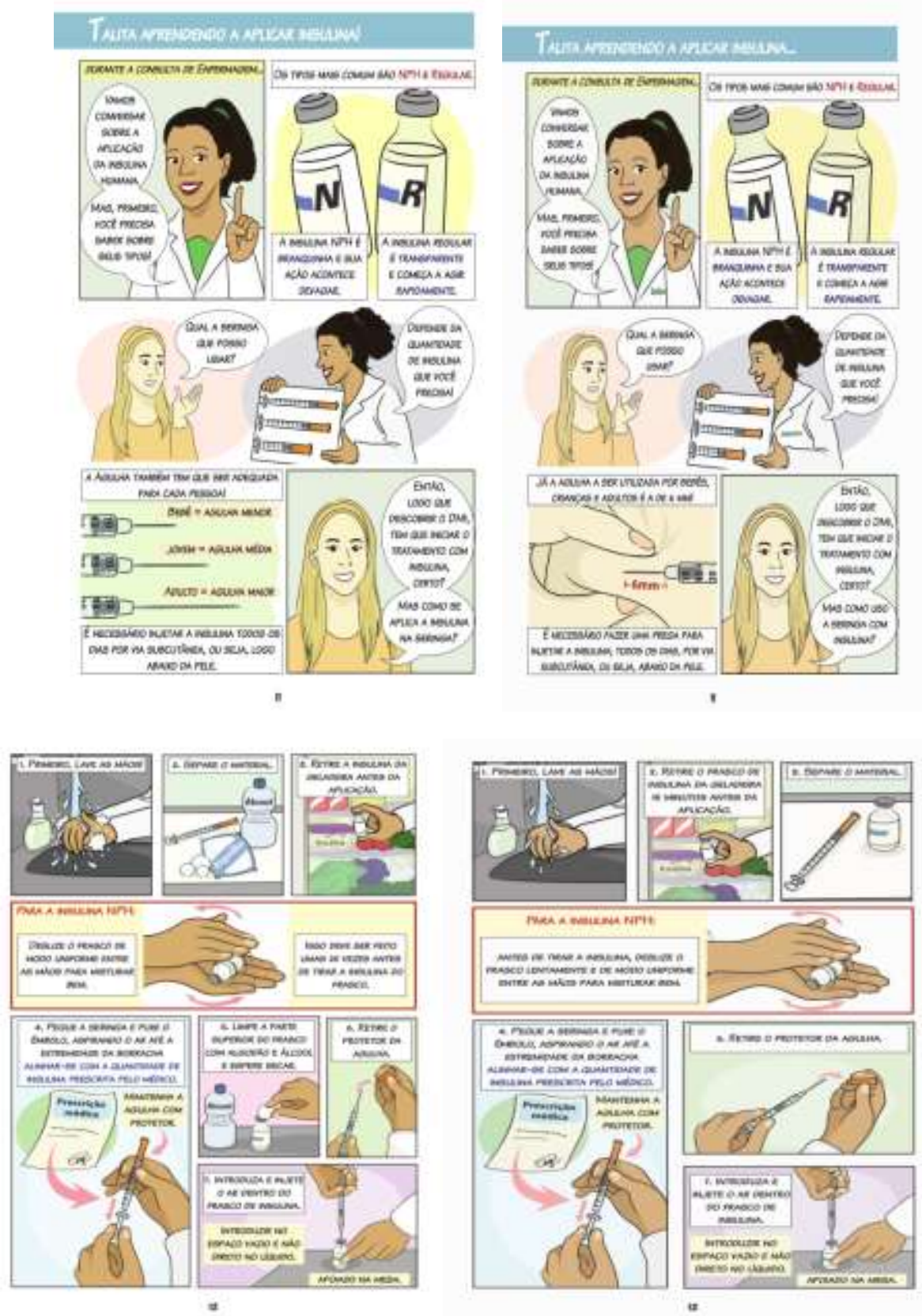

Fonte: Autoria própria: Rebouças et al, (2016) - Tecnologia educativa: "Diabetes não é brinquedo! - História em quadrinhos para adolescente." Elaborada em 2016.

Nas páginas 11 e 12 (Figura 5), observa-se a enfermeira Bete ensinando e detalhando à Talita a forma correta de aplicar a insulina. Uma juíza técnica sugeriu que modificasse esse quadro que tem agulha específica para bebê, jovens e adultos, pois todos devem usar agulha de $6 \mathrm{~mm}$ e fazer prega cutânea. No último quadrinho da página, a personagem Talita finaliza com a indagação, "Mas como se aplica a insulina na seringa?" que foi corrigido por, "Mas como uso a seringa com insulina?".

Após validação, uma juíza sugeriu que retirasse o álcool dos materiais. Além disso, juíza técnica solicitou que o frasco de insulina fosse retirado 15 minutos antes da aplicação e esse frasco deve ser deslizado lentamente.

A próxima Figura (6), é uma continuação da explicação da aplicação de insulina, sendo nessas imagens, focadas na 
aplicação no paciente.

Figura 6: Versão pré-validação e pós-validação da continuação da forma correta de aplicação da insulina nos pacientes com DM tipo 1 na história em quadrinhos.
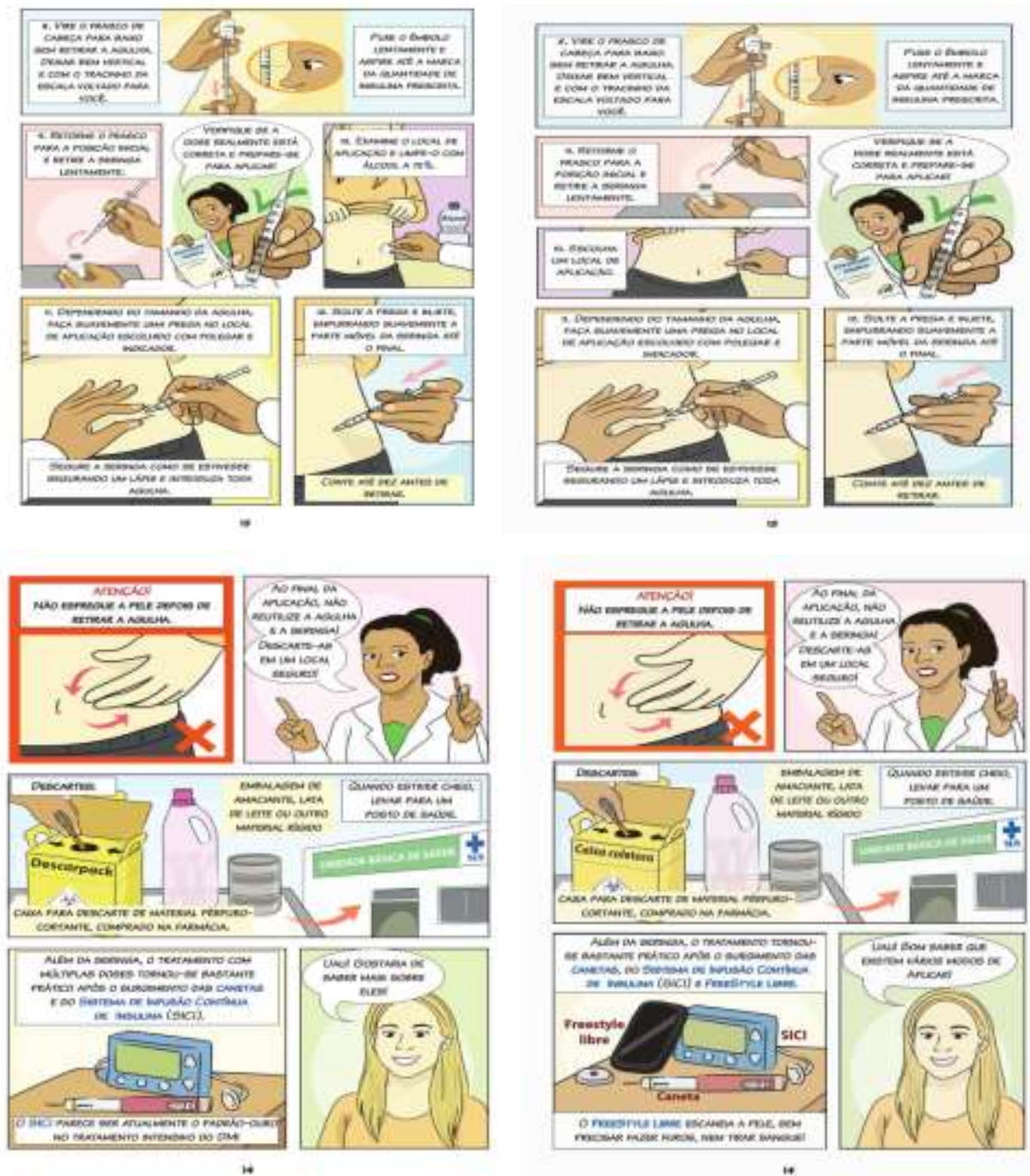

Fonte: Autoria própria: Rebouças et al, (2016) - Tecnologia educativa: "Diabetes não é brinquedo! - História em quadrinhos para adolescente." Elaborada em 2016.

Na Figura 6, observa-se a continuação da explicação do modo correto de aplicar a insulina no paciente. Na página 13, explica os passos para administração da insulina com seringa. Após a validação, uma juíza sugeriu que retirasse o uso de álcool para limpeza da pele.

Na página 14 (Figura 6), houve orientação em relação ao descarte de material perfuro-cortante. Após a validação, o nome "descarpack" foi substituído por "caixa coletora", pois esse seria o nome de marca. Como foi abordado o SICI, uma juíza sugeriu falar brevemente sobre o sensor de monitoramento da glicemia que não necessita de agulhas ou lancetas para verificação da glicemia. O FreeStyle Libre é um monitor de glicose vendido no Brasil que funciona sem necessidade de fazer furos na pele e retirar sangue. Dispensa esse uso de lancetas, pois realiza o escaneamento da pele. O aparelho é caro para a realidade de muitas famílias, mas achou-se necessário divulgar para que os adolescentes busquem melhores condições de manejo do DM1. Retirado quadrinho em que descreve que SICI é padrão ouro. 
Uma juíza sugeriu que acrescentasse que não precisa guardar em geladeira. Modificado também o nome "descarpack" por caixa coletora. Descarte a agulha nos locais já mencionados já mencionados anteriormente" foi substituído "nos locais mencionados anteriormente."

$\mathrm{Na}$ temática "Talita aprendendo os locais de aplicação" (Figura 7), uma juíza sugeriu a que evitasse colocar informações entre parênteses, pois são as falas das personagens. Então, na fala da enfermeira Bete tinha lipodistrofia entre parênteses. Não há recomendação de lavar as mãos com sabonete. Após a validação, mantido só a agulha de 6mm. Retirado no adulto o ângulo de $90^{\circ}$.

Figura 7: Versão pré-validação e pós-validação da temática "Locais de aplicação" da insulina do paciente com DM tipo 1 na história em quadrinhos.
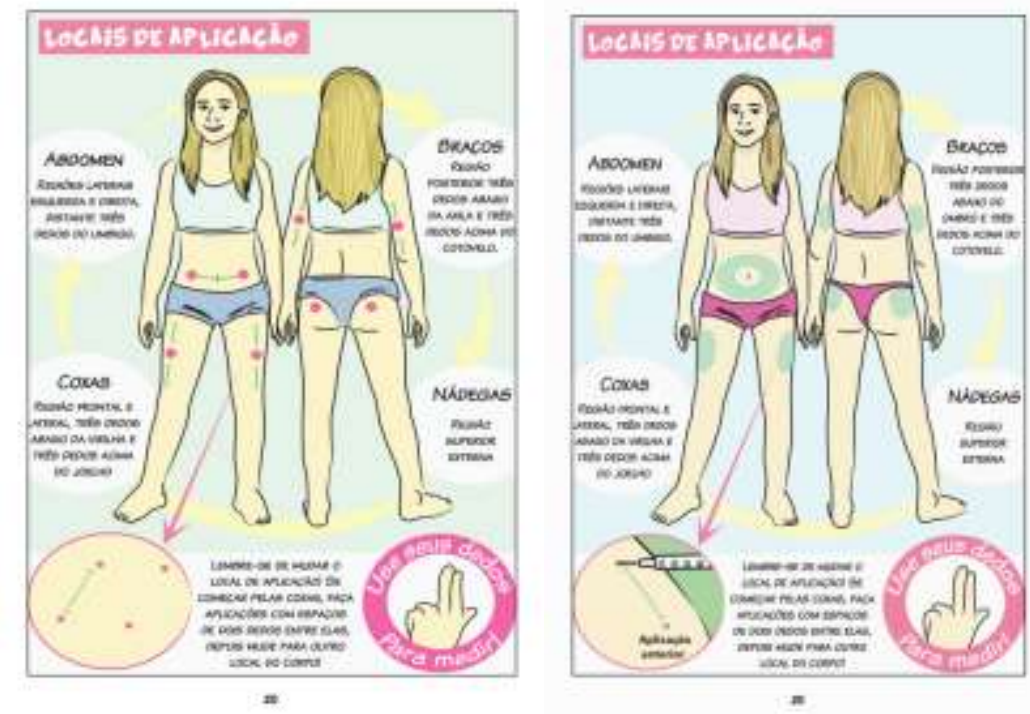

Fonte: Autoria própria: Rebouças et al, (2016) - Tecnologia educativa: "Diabetes não é brinquedo! - História em quadrinhos para adolescente." Elaborada em 2016.

Na Figura 7, é possível visualizar os locais corretos de aplicação da insulina nos pacientes com DM tipo 1. Nesta temática, os juízes sugeriram que no braço deve ser 3 dedos abaixo do ombro, não na axila. A região de aplicação da insulina nas nádegas ficou incorreta. Corrigida a imagem de acordo com a Sociedade Brasileira de Diabetes, destacando o quadrante superior externo e o no local no meio das nádegas.

Na temática "Talita x Dieta", os juízes sugeriram que fosse substituído por "Talita e a dieta...” sugerido evitar pontos de interrogação ou exclamação nos títulos e substituir por reticências. Durante as entrevistas os adolescentes citaram xilito como alimento que mesmo ciente que não é adequado, mas é difícil resistir. As perguntas da atividade educativa foram as do roteiro de entrevistas aplicado pela pesquisadora e as respostas dos adolescentes.

No item seis do roteiro norteador ("Sugira figuras ou imagens que poderiam ser acrescentadas na história em quadrinhos"), o piquenique também foi sugestão de um adolescente. Uma juíza comentou que termo xilito citado na versão inicial denominação bastante utilizado no Ceará, mas em outras regiões é conhecido como salgadinho de milho. Incluiu-se também ilustrações de tapioca e de cuscuz na história em quadrinhos para valorizar os alimentos saudáveis da cultura nordestina.

Nos últimos tópicos da história em quadrinhos, cujos títulos eram "Talita Fitness" e "Dicas de Exercício", após a validação, foi retirado o ponto de exclamação conforme sugestão de uma juíza. No quadro, "Um mês depois, Talita teve alta hospitalar e começou a praticar exercício" foi substituído por "Logo depois, Talita começou a praticar exercícios", pois o 
tópico "Talita na internação devido à hipoglicemia" foi substituído por "Talita aprendendo contagem de carboidrato...”. Incluso outro personagem para ilustrar os amigos da Talita nomeado aleatoriamente de Bento (Figura 8).

Figura 8: Versão pré-validação e pós-validação da temática mudança de hábitos no controle da DM tipo 1 na história em quadrinhos.
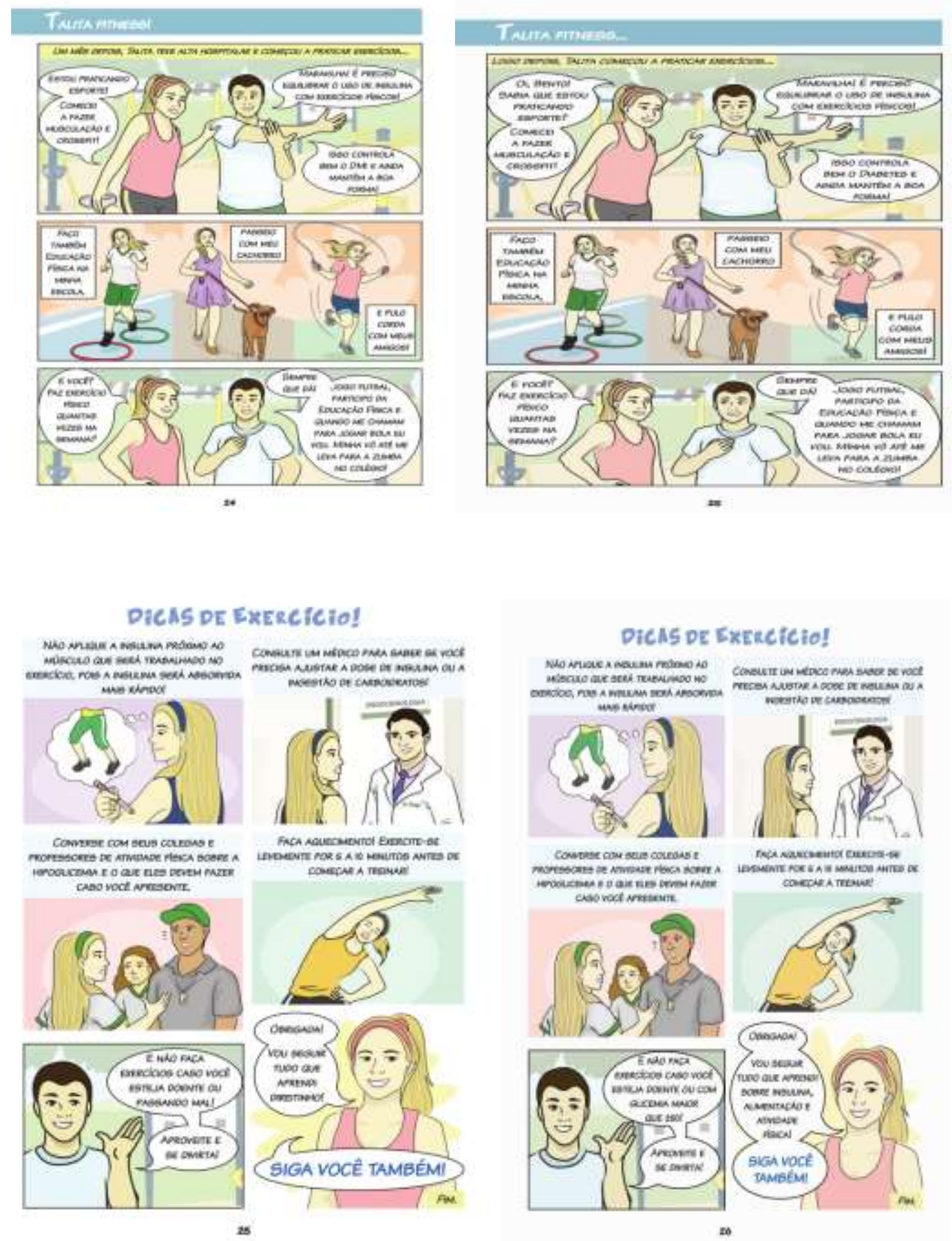

Fonte: Autoria própria: Rebouças et al, (2016) - Tecnologia educativa: "Diabetes não é brinquedo! - História em quadrinhos para adolescente." Elaborada em 2016.

O uso de Tecnologias Educativas aplicadas no âmbito da saúde, constituem instrumentos relevantes no processo de cuidar, facilitando o diálogo entre o paciente e os profissionais de saúde (Albuquerque et al, 2016).

Por meio do uso dos manuais educativos, é possível tornar mais eficiente o trabalho da equipe multiprofissional na 
orientação do paciente e de seus familiares. Além disso, percebe-se que o uso de materiais educativos, é possível favorecer uma visão centrada no autocuidado e na corresponsabilização do paciente (Echer, 2005).

$\mathrm{Na}$ presente pesquisa, foi possível observar que de uma forma geral, os juízes classificaram a história em quadrinho um material adequado para o uso na prática clínica. Vale ressaltar, que embora as imagens agreguem um maior valor atrativo ao material, é fundamental a observação da adequação da linguagem empregada. É por meio dela que o material se tornará compreensível pelo público-alvo, e possam apresentar relevância prática (Pinto et al, 2018).

Após a construção do material, percebe-se que é na avaliação por profissionais de diferentes áreas em que de fato, se observa o trabalho da equipe, com a valorização de uma visão mais ampla e de abordagens diversas sobre um mesmo assunto (Echer, 2005). Outro ponto relevante proporcionado pela validação por especialistas é tornar o material mais aplicável na realidade dos pacientes (Chaves et al, 2021).

A tecnologia educativa apresenta uma relevância história em quadrinhos, tendo como predominância a sistematização de planejar, implementar e avaliar a instrução nas pesquisas de aprendizagem humana, elaboração comunicativa, simples e lúdica dos materiais educativos (Luckesi, 1986). Deve-se salientar sobre a importância do material educativo que circula no contexto do cuidar- educando, tendo como pilar o rigor do processo de avaliação e validação por juízes especialistas e pelo público-alvo antes de ser aplicado.

A educação em saúde visa propiciar cuidado emancipatório, ou seja, capacitar o indivíduo para o autocuidado. No que concerne à educação infantil, destacam-se os pressupostos do Construtivismo (Piaget, 1967). Para tanto, incentivar a construção do conhecimento e a participação ativa do adolescente através do diálogo, o estímulo à dúvida e o desenvolvimento do raciocínio, torna-se um aliado no processo ensino-aprendizagem, tanto para a adolescente como promove a qualidade da atuação durante $o$ atendimento de uma equipe multiprofissional.

\section{Considerações Finais}

O desenvolvimento desta tecnologia educativa foi realizado em um processo dialógico e coletivo. O conteúdo obtido durante as entrevistas foi utilizado na construção da história em quadrinhos. Para melhor eficácia do material, realizou-se avaliação de juízes especialistas de conteúdo técnicos e de propaganda e marketing.

A participação dos juízes possibilitou a adequação e o aprimoramento da tecnologia, pois as sugestões destes profissionais foram valiosas para o aperfeiçoamento do material, de modo a assegurar ao instrumento melhor eficácia. Os itens abordados na história em quadrinhos foram principalmente a monitorização glicêmica, insulina, dieta e exercício. Acrescentado contagem de carboidrato após validação. O material é uma estratégia educativa de promoção da saúde através de uma linguagem lúdica.

Ademais o estudo apresentou limitação durante a identificação de artigos que correlacionassem a DM tipo 1 com o ser adolescente. Enfatiza-se que o processo de validação e construção se deu no ano de 2016, sendo esta primeira edição da cartilha com conteúdo baseado em publicações anteriores a esse ano. Desta forma, uma nova edição será realizada com as modificações feitas nas Diretrizes da Sociedade Brasileira de Diabetes: 2019-2020. Porém, a pesquisa apresenta uma importância assertiva para a tecnologia educativa, por se tratar de uma ferramenta pedagógica de educação em saúde com linguagem simples, lúdica e que proporcionará melhoria na adesão dos adolescentes com DM tipo 1. Além disso, a estratégia educativa, fortalece o vínculo e a confiança do profissional com o adolescente, pois promove a construção do conhecimento e a participação ativa do adolescente e concomitantemente melhora a adesão ao tratamento. 


\section{Referências}

Albuquerque, A. F. L. L., et al. (2016). Tecnologia para o autocuidado da saúde sexual e reprodutiva de mulheres estomizadas. Rev. Bras. Enferm, 69(6), 1164-71.

Alexandre, N. M. C., \& Coluci, M. Z. (2011). O. Validade de conteúdo nos processos de construção e adaptação de instrumentos de medidas. Ciência \& Saúde Coletiva, 16 (7), 3061-3068.

Bardin, L. (2011). Análise de conteúdo. Edições 70.

BRASIL. Resolução 466/2012. (2013). Diretrizes e normas regulamentadoras de pesquisas envolvendo seres humanos. Ministério da Saúde/Conselho Nacional de Saúde, Brasília.

Chaves, M. A. A., et al. (2021). Elaboração e validação de um álbum seriado para prevenção do pé diabético. Revista Cuidarte, $12(1)$ : e1233.

Dewes, J. O. (2013). Amostragem em Bola de Neve e Respondent - Driven Sampling: uma descrição de métodos. Monografia - Universidade Federal do Rio Grande do Sul, Porto Alegre, Rio Grande do Sul, Brasil.

Dodt, R. C. M. (2011) Elaboração e validação de tecnologia educativa para autoeficácia da amamentação. Tese de Doutorado - Faculdade de Farmácia, Odontologia e Enfermagem, Universidade Federal do Ceará, Fortaleza, Ceará, Brasil.

Echer, I. C. (2005). Elaboração de manuais de orientação para o cuidado em saúde. Rev. Latino-Am. Enfermagem, $13(5), 754-757$.

Haller, M. J., Atkinson, M. A., \& Schatz. (2005). D. Type 1 diabetes mellitus: etiology, presentation and management. Pediatr. Clin. North Am., 52, 15531578 .

International Diabetes Federation (IDF) (2019). IDF Diabetes Atlas. International Diabetes Federation.

Katsarou, A., et al. (2017). Type 1 diabetes mellitus. Nat Rev Dis Primers, 3(17016), 1-17.

Lima Filho, B., et al. (2020). Internações por Diabetes Mellitus em idosos brasileiros e suas implicações regionais nos últimos 10 anos. Research, Society and Development, 9(8), e40985106.

Luckesi, C. C. (1986) Independência e inovação em tecnologia educacional: ação-reflexão. Tecnologia Educacional, 15(71/72), 55-64.

Luna, R., et al. (2005). The role of diabetes duration, puberal development and metabolic control in growth in children with type 1 diabetes mellitus. $J$ Pediatr Endocrinol Metab., 18(12), 1425-1431.

Mallmann, D. G., et al. (2015). Educação em Saúde como principal alternativa para promover a saúde do idoso. Ciência \& Saúde Coletiva, $20(6)$, 1763-72.

Massara, C. L., et al. (2016). Caracterização de materiais educativos impressos sobre esquistossomose, utilizados para educação em saúde em áreas endêmicas no Brasil. Epidemio. Serv. Saúde, 25(3), 575-84.

Ministério da Saúde. Secretaria de Atenção à Saúde. Departamento de Atenção Básica. Diabetes Mellitus. (2013). Caderno de Atenção Básica. Brasília: Ministério da Saúde, 2013.

Nascimento, M. H. M. (2012). Tecnologia para medir o cuidar - educando no acolhimento de "familiares cangurus" em unidade neonatal: Estudo de Validação. Dissertação - Mestrado Associado de Enfermagem UEPA - UFAM) - Universidade Estadual do Pará, Belém, Pará, Brasil.

Nilson, E. A. F., et al. (2020) Custos atribuíveis a obesidade, hipertensão e diabetes no Sistema Único de Saúde, Brasil, 2018. Revista Panamericana de Salud Pública, 44, e32.

Orbey, N. C., et al. (2007). The influence of dietary intake and meal pattern on blood glucose control in children and adolescentes using intensive insulin treatment. Diabetologia., 50 (10), 2044-2051.

Pace, A. E., Nunes, P. D., \& Ochoa-Vigo, K. (2003). O conhecimento dos familiares a cerca da problemática do portador de diabetes mellitus. Rev. Latino-Am. Enf., 11(3), 312-319.

Pasquali, L. (1997). Psicometria: teoria e aplicações. UnB.

Piaget, J. (1967). O raciocínio na criança. Record.

Polit, D. F., \& Beck, C. T. (2011). Fundamentos de pesquisa em enfermagem: avaliação de evidências para a prática de enfermagem. Artmed.

Pinto, S. de L., et al. (2018). Posicionamento do paciente para raquianestesia: construção e validação de álbum seriado. Acta Paulista de Enfermagem, 31(1), $25-31$.

Queiroz, K. C., Silva, I. N., \& Alfenas, R. C. G. (2010). Associação entre fatores nutricionais e o controle glicêmico de crianças e adolescentes com diabetes melito tipo 1. Arq Bras Endocrinol Metab., 54 (3), 319-25.

Salci, M. A., Meirelles, B. H. S., \& Silva, D. M. G. V. da. (2018). Educação em saúde para prevenção das complicações crônicas do diabetes mellitus na atenção primária. Escola Anna Nery, 22 (1), e20170262.

Salin, A. B., et al. (2019). Diabetes Mellitus tipo 2: perfil populacional e fatores associados à adesão terapêutica em Unidades Básicas de Saúde em Porto Velho-RO. Revista Eletrônica Acervo Saúde, 33, e1257-e1257. 
Research, Society and Development, v. 10, n. 5, e34710515087, 2021

(CC BY 4.0) | ISSN 2525-3409 | DOI: http://dx.doi.org/10.33448/rsd-v10i5.15087

Sociedade Brasileira de Diabetes (2015). Diretrizes da Sociedade Brasileira de Diabetes: 2014-2015. AC Farmaceutica.

Sociedade Brasileira de Diabetes (2019). Diretrizes da Sociedade Brasileira de Diabetes: 2019-2020. AC Farmacêutica.

Teixeira, E., Nascimento, M. H. M., \& Medeiros, H. P. (2014). Referênciais metodológicos para validação de tecnologias cuidativo -educacionais. In: Nietsche, E. A., Teixeira, E., Medeiros, H. P., (Orgs.). Tecnologias cuidativo-educacionais: uma possibilidade para o empoderamento do(a) enfermeiro(a). Moriá Editora.

World Health Organization.(2015). Adolescent health. http://www.who.int/topics/adolescent_health/en> 